\title{
Peri-conceptional diet patterns and risk of gestational diabetes mellitus in South Indian women
}

\author{
A. Mahendra ${ }^{1,2}$, S.H. Kehoe ${ }^{1}$, S.R. Crozier ${ }^{1}$, K. Kumaran ${ }^{1,2}$, G.V. Krishnaveni ${ }^{2}$, N. Arun ${ }^{2}$, \\ Padmaja $^{3}$, P. Kini ${ }^{4}$, U. Taskeen ${ }^{5}$, Chaitra ${ }^{6}$, K.T. Kombanda ${ }^{7}$, M. Johnson ${ }^{1}$, C. Osmond ${ }^{1}$ and \\ C.H.D. Fall ${ }^{1}$ \\ ${ }^{1}$ MRC Lifecourse Epidemiology Unit, University of Southampton, Southampton, UK, \\ ${ }^{2}$ Epidemiology Research Unit, CSI Holdsworth Memorial Hospital, Mysore, India, \\ ${ }^{3}$ Department of Obstetrics and Gynaecology, Bangalore Baptist Hospital, Bangalore, India, \\ ${ }^{4}$ Department of Obstetrics and Gynaecology, Cloudnine Hospital, Bangalore, India, \\ ${ }^{5}$ Previously affiliated to Epidemiology Research Unit, CSI Holdsworth Memorial Hospital, Mysore, India, \\ ${ }^{6}$ Department of Dietetics, Cloudnine Hospital, Bangalore, India and \\ ${ }^{7}$ Institute for Physical Activity and Nutrition (IPAN), Deakin University, Geelong, Australia
}

Gestational diabetes mellitus (GDM) affects $20 \%$ of pregnancies in India ${ }^{(1)}$. In Western countries healthy diet patterns characterised by wholegrains, fruits and vegetables are associated with a lower GDM risk; and unhealthy patterns (refined grains, fried and fast food, high sugar, red and processed meat) with higher risk. Evidence from low- and middle-income countries is sparse, and diet pattern-GDM associations in India have not been explored. We aimed to identify peri-conceptional diet patterns among women in Bangalore and examine their associations with GDM risk.

BANGLES (BAngalore Nutrition Gestational diabetes LifEstyle Study) started in 2016, a prospective observational study in which pregnant women $(n=785)$ of varied socio-economic status were recruited at 5-16 weeks' gestation. Peri-conceptional diet was assessed retrospectively at recruitment, using a validated 224-item Food Frequency Questionnaire (FFQ). The 224 FFQ foods were reduced to 68 food-groups as input variables for principal component analysis to identify diet patterns. GDM was defined by a 75-gram Oral Glucose Tolerance Test (OGTT) at 24-28 weeks' gestation, applying WHO 2013 criteria. Diet pattern-GDM associations were analysed using multivariate logistic regression adjusting for 'a priori' confounders.

GDM prevalence was $22 \%$. Three standardised distinct peri-conceptional diet patterns were identified: a) High-diversity, urban (HDU), characterised by consumption of a diversity of expensive, home-cooked, processed, healthy and unhealthy foods including wholegrains, fruits, vegetables, dairy, nuts, seeds, egg, poultry, meat, fast-food and sweets was associated with older, affluent, more educated and urban women; b) Rice-fried snacks-chicken-sweets (RFCS) pattern, characterised by low diet-diversity, was associated with younger, thinner, less educated women from lower-income, rural, joint families; c) Healthy, traditional vegetarian (HTV) pattern, characterised by home-cooked, vegetarian and non-processed foods was associated with women being thinner, less educated, affluent from rural and joint families. The HDU pattern was associated with a lower GDM risk (aOR: 0.80 per SD, 95\% CI: 0.64 , $0.99, \mathrm{p}=0.04)$ after adjusting for confounders, not significant after correction for multiple testing. Women's BMI was the strongest risk factor for GDM and possibly partly mediated diet-GDM associations.

The findings support global recommendations to encourage women to attain a healthy pre-pregnancy BMI. The HDU patternlower GDM association, although not significant, was consistent with national and global diet recommendations to increase diet diversity ${ }^{(2,3 \& 4)}$. However, the HDU and RFCS patterns consisting of healthy and unhealthy foods may indicate low awareness and the need to invest in public education about healthy/unhealthy foods. Higher socio-economic status was positively associated with diet diversity (HDU \& HTV). This highlights the need for national policies to make wholegrains, fruits, vegetables, dairy and poultry foods more affordable ${ }^{(3)}$. In the future, the construction of a healthy diet index may be useful.

\section{Acknowledgements}

We thank the study participants and staff of Bangalore Baptist and Cloudnine Hospitals for their support to the study. BANGLES was funded by the Schlumberger Foundation as a doctoral Faculty for the Future Fellowship awarded to AM.

\section{References}

1. Li KT, Naik S, Alexander M, et al. (2018) Acta Diabetol 55(6), 613-625.

2. World Health Organization (1990) WHO Technical series 797, 49-50.

3. Willett W, Rockström J \& Loken B (2019) Lancet 393, 447-492.

4. What India eats report (2020), Govt. of India, 2020. [Ävailable at: https://www.nin.res.in/nutrition2020/what_india_eats.pdf] 